\title{
New characterizations for the products of differentiation and composition operators between Bloch-type spaces
}

Yu-Xia Liang ${ }^{1}$ and Xing-Tang Dong ${ }^{2^{*}}$

"Correspondence:

dongxingtang@163.com

${ }^{2}$ Department of Mathematics,

Tianjin University, Tianjin, 300072

P.R. China

Full list of author information is

available at the end of the article

\begin{abstract}
We use a brief way to give various equivalent characterizations for the boundedness and the essential norm of the operator $C_{\varphi} D^{m}$ acting on Bloch-type spaces. At the same time, we use this method to easily get a known characterization for the operator $D C_{\varphi}$ on Bloch-type spaces.

MSC: Primary 47B38; secondary 26A24; 32H02; 47B33
\end{abstract}

Keywords: essential norm; differentiation; composition operator; Bloch-type space

\section{Introduction and preliminaries}

Recently there has been a considerable interest on various product-type operators (see, e.g. [1-19]), and among them on products of composition and differentiation operators (see, e.g. $[1,2,4,5,7-12,15-19])$. One of the problems of interest is to characterize the boundedness and compactness of the composition operator $C_{\varphi}$ acting on Bloch-type spaces in terms of the $n$th power of the analytic self-mapping $\varphi$ of the unit disk $\mathbb{D}$. Very recently, the first author and Zhou have given the characterizations for the boundedness and the essential norm of the products of differentiation and composition operator $C_{\varphi} D^{m}$ and $D C_{\varphi}$ acting on Bloch-type spaces in $[9,10]$, respectively. Inspired by [20], we present here an easier way to research the corresponding problem. Moreover, by this brief method, we first give new equivalent characterizations for the boundedness and the essential norm of the operator $C_{\varphi} D^{m}$, and then we obtain the same results for the operator $D C_{\varphi}$ as in the paper [10].

Let $\mathbb{D}$ denote the unit disk in the complex plane $\mathbb{C}$. Denote $H(\mathbb{D})$ the space of all holomorphic functions on $\mathbb{D}$ and $S(\mathbb{D})$ the collection of all holomorphic self-mappings on $\mathbb{D}$. The composition operator $C_{\varphi}$ is defined by $C_{\varphi} f=f \circ \varphi$ for $f \in H(\mathbb{D})$ and $\varphi \in S(\mathbb{D})$.

The Bloch space of $v$-type

$$
\mathcal{B}_{v}=\left\{f \in H(\mathbb{D}):\|f\|_{\mathcal{B}_{v}}=\sup _{z \in \mathbb{D}} v(z)\left|f^{\prime}(z)\right|<\infty\right\}
$$

is a Banach space endowed with the norm $|f(0)|+\|f\|_{\mathcal{B}_{v}}$, where the weight $v: \mathbb{D} \rightarrow \mathbb{R}_{+}$is a continuous, strictly positive and bounded function.

@2014 Liang and Dong; licensee Springer. This is an Open Access article distributed under the terms of the Creative Commons Attribution License (http://creativecommons.org/licenses/by/2.0), which permits unrestricted use, distribution, and reproduction in any medium, provided the original work is properly cited. 
For the standard weights $v_{\alpha}(z)=\left(1-|z|^{2}\right)^{\alpha}$ for $\alpha>0$, we denote $\mathcal{B}_{v}=\mathcal{B}^{\alpha}$ and

$$
\|f\|_{\alpha}=\sup _{z \in \mathbb{D}}\left(1-|z|^{2}\right)^{\alpha}\left|f^{\prime}(z)\right|
$$

Similarly, $\mathcal{B}^{\alpha}$ is a Banach space under the norm $\|f\|_{\mathcal{B}^{\alpha}}=|f(0)|+\|f\|_{\alpha}$. When $\alpha=1$, we get the classical Bloch space $\mathcal{B}$. We refer the readers to the book [21] for more information as regards the above spaces.

The weighted Banach space of analytic functions

$$
H_{v}^{\infty}=\left\{f \in H(\mathbb{D}):\|f\|_{\nu}=\sup _{z \in \mathbb{D}} v(z)|f(z)|<\infty\right\}
$$

is a Banach space endowed with the norm $\|\cdot\|_{\nu}$. The weight $v$ is called radial, if $v(z)=v(|z|)$ for all $z \in \mathbb{D}$. For a weight $v$, the associated weight $\tilde{v}(z)$ is defined by

$$
\tilde{v}(z)=\left(\sup \left\{|f(z)|: f \in H_{v}^{\infty},\|f\|_{v} \leq 1\right\}\right)^{-1}, \quad z \in \mathbb{D} .
$$

For the standard weights $v_{\alpha}(z)=\left(1-|z|^{2}\right)^{\alpha}(0<\alpha<\infty)$, we have $\tilde{v}_{\alpha}(z)=v_{\alpha}(z)$. We refer the interested readers to [22, p.39]. In this case, we denote $H_{\nu}=H_{v_{\alpha}}^{\infty}$ and

$$
\|f\|_{v_{\alpha}}=\sup _{z \in \mathbb{D}}\left(1-|z|^{2}\right)^{\alpha}|f(z)|
$$

Then $H_{v_{\alpha}}^{\infty}$ is a Banach space under the norm $\|f\|_{\nu_{\alpha}}$.

For $\varphi \in S(\mathbb{D}), u \in H(\mathbb{D})$, the weighted composition operator $u C_{\varphi}$ is defined by

$$
u C_{\varphi}(f)=u \cdot(f \circ \varphi), \quad f \in H(\mathbb{D})
$$

As for $u \equiv 1$, the weighted composition operator is the usual composition operator $C_{\varphi}$. When $\varphi$ is the identity mapping $I$, the operator $u C_{I}$ is the multiplication operator $M_{u}$.

The differentiation operator $D$ is defined by

$$
D f=f^{\prime}, \quad f \in H(\mathbb{D}) .
$$

The products of differentiation and composition operators $D C_{\varphi}$ and $C_{\varphi} D^{m}$ are defined, respectively, as follows:

$$
D C_{\varphi} f(z)=f^{\prime}(\varphi(z)) \varphi^{\prime}(z), \quad C_{\varphi} D^{m} f=f^{(m)} \circ \varphi, \quad f \in H(\mathbb{D}), m \in \mathbb{N} .
$$

The essential norm of a continuous linear operator $T$ between two normed linear spaces $X$ and $Y$ is its distance from the compact operators. That is,

$$
\|T\|_{e, X \rightarrow Y}=\inf \{\|T-K\|: K \text { is compact }\},
$$

where $\|\cdot\|$ denotes the operator norm. Notice that $\|T\|_{e, X \rightarrow Y}=0$ if and only if $T$ is compact, so the estimate on $\|T\|_{e, X \rightarrow Y}$ will lead to the condition for the operator $T$ to be compact. 
Throughout this paper, $C$ will denote a positive constant, the exact value of which will vary from one appearance to the next. The notations $A \asymp B, A \preceq B, A \succeq B$ mean that there maybe different positive constants $C$ such that $B / C \leq A \leq C B, A \leq C B, A \geq C B$.

For convenience of the reader we list the results related with our conclusions in this paper.

Theorem A [9, Theorem 1] Let $0<\alpha, \beta<\infty, m$ be a nonnegative integer and $\varphi$ be a holomorphic self-map of the unit disk $\mathbb{D}$. Then $C_{\varphi} D^{m}: \mathcal{B}^{\alpha} \rightarrow \mathcal{B}^{\beta}$ is bounded if and only if

$$
\sup _{n \in \mathbb{N}} n^{\alpha-1}\left\|C_{\varphi} D^{m} I_{n}(z)\right\|_{\beta}<\infty .
$$

Theorem B [9, Theorem 2] Let $0<\alpha, \beta<\infty, m$ be a nonnegative integer and $\varphi$ be a holomorphic self-map of the unit disk $\mathbb{D}$. Suppose that $C_{\varphi} D^{m}: \mathcal{B}^{\alpha} \rightarrow \mathcal{B}^{\beta}$ is bounded. Then the estimate for the essential norm of $C_{\varphi} D^{m}: \mathcal{B}^{\alpha} \rightarrow \mathcal{B}^{\beta}$ is

$$
\left\|C_{\varphi} D^{m}\right\|_{e} \asymp \limsup _{n \rightarrow \infty} n^{\alpha-1}\left\|C_{\varphi} D^{m} I_{n}(z)\right\|_{\beta}
$$

where $I_{n}(z)=z^{n}, z \in \mathbb{D}, n \in \mathbb{N}$.

Theorem C [10, Theorem 2.3] Let $0<\alpha, \beta<\infty$, and $\varphi \in S(\mathbb{D})$. Then $D C_{\varphi}: \mathcal{B}^{\alpha} \rightarrow \mathcal{B}^{\beta}$ is bounded if and only if

$$
\sup _{n \geq 1} n^{\alpha}\left\|I_{\varphi^{\prime}}\left(\varphi^{n}\right)\right\|_{\beta}<\infty \quad \text { and } \quad \sup _{n \geq 1} n^{\alpha}\left\|J_{\varphi^{\prime}}\left(\varphi^{n-1}\right)\right\|_{\beta}<\infty
$$

Theorem D [10, Theorem 3.5] Let $0<\alpha, \beta<\infty$ and $\varphi \in S(\mathbb{D})$. Suppose that $D C_{\varphi}: \mathcal{B}^{\alpha} \rightarrow$ $\mathcal{B}^{\beta}$ is bounded. Then the estimate for the essential norm of $D C_{\varphi}: \mathcal{B}^{\alpha} \rightarrow \mathcal{B}^{\beta}$ is

$$
\max \left\{\frac{A}{3 \cdot 2^{\alpha+1}}, \frac{B}{2^{\alpha+1}(3 \alpha+4)}\right\} \leq\left\|D C_{\varphi}\right\|_{e} \preceq(A+B),
$$

where $A:=\left(\frac{e}{2(\alpha+1)}\right)^{\alpha+1} \lim \sup _{n \rightarrow \infty} n^{\alpha}\left\|I_{\varphi^{\prime}}\left(\varphi^{n}\right)\right\|_{\beta}$ and $B:=\left(\frac{e}{2 \alpha}\right)^{\alpha} \lim \sup _{n \rightarrow \infty} n^{\alpha}\left\|J_{\varphi^{\prime}}\left(\varphi^{n-1}\right)\right\|_{\beta}$. The definitions of $I_{\varphi^{\prime}}\left(\varphi^{n}\right)$ and $J_{\varphi^{\prime}}\left(\varphi^{n-1}\right)$ can be found in Section 4.

We would like to point out that the first author and Zhou got the above four theorems by using complex calculations and intricate discussions. In this paper, we will use a brief way to give other equivalent characterizations for the boundedness and the essential norm of $C_{\varphi} D^{m}: \mathcal{B}^{\alpha} \rightarrow \mathcal{B}^{\beta}$ on the unit disk in Section 3. In addition, using this method we will show new proofs of Theorem $C$ and Theorem D in Section 4.

\section{Lemmas}

In this section we quote some lemmas for our further application. The first lemma is a well-known characterization for $\mathcal{B}^{\alpha}(0<\alpha<\infty)$.

Lemma 2.1 For $f \in H(\mathbb{D}), m \in \mathbb{N}$ and $\alpha>0$. Then

$$
f \in \mathcal{B}^{\alpha} \quad \Leftrightarrow \quad\|f\|_{\alpha} \asymp \sum_{j=0}^{m-1}\left|f^{(j)}(0)\right|+\sup _{z \in \mathbb{D}}\left(1-|z|^{2}\right)^{\alpha+m-1}\left|f^{(m)}(z)\right|<\infty .
$$


So for $f \in \mathcal{B}^{\alpha}$, the above lemma implies that $f^{\prime} \in H_{\nu_{\alpha}}^{\infty}$ and more general $f^{(m+1)} \in H_{\nu_{\alpha+m}}^{\infty}$. Therefore, theories of the weighted composition operator $u C_{\varphi}: H_{v}^{\infty} \rightarrow H_{w}^{\infty}$ play a key role in the proof of our main results. Here we list some lemmas which will be used later.

Lemma 2.2 [23, Proposition 3.1] Let $v$ and $w$ be weights. Then the weighted composition operator $u C_{\varphi}: H_{v}^{\infty} \rightarrow H_{w}^{\infty}$ is bounded if and only if

$$
\sup _{z \in \mathbb{D}} \frac{w(z)|u(z)|}{\tilde{v}(\varphi(z))}<\infty
$$

Moreover, the following holds:

$$
\left\|u C_{\varphi}\right\|_{H_{v}^{\infty} \rightarrow H_{w}^{\infty}}=\sup _{z \in \mathbb{D}} \frac{w(z)|u(z)|}{\tilde{v}(\varphi(z))} .
$$

Lemma 2.3 [23, Theorem 4.4] Let $v$ and $w$ be radial, non-increasing weights tending to zero at the boundary of $\mathbb{D}$. Suppose $u C_{\varphi}: H_{\nu}^{\infty} \rightarrow H_{w}^{\infty}$ is bounded. Then

$$
\left\|u C_{\varphi}\right\|_{e, H_{v}^{\infty} \rightarrow H_{w}^{\infty}} \asymp \lim _{r \rightarrow 1} \sup _{|\varphi(z)|>r} \frac{w(z)|u(z)|}{\tilde{v}(\varphi(z))} .
$$

Lemma 2.4 [24, Theorem 2.4] Let $v$ and $w$ be radial, non-increasing weights tending to zero at the boundary of $\mathbb{D}$. Then

(a) $u C_{\varphi}: H_{v}^{\infty} \rightarrow H_{w}^{\infty}$ is bounded if and only if

$$
\sup _{n \geq 0} \frac{\left\|u \varphi^{n}\right\|_{w}}{\left\|z^{n}\right\|_{v}}<\infty
$$

with the norm comparable to the above supremum.

(b) $\left\|u C_{\varphi}\right\|_{e, H_{v}^{\infty} \rightarrow H_{w}^{\infty}}=\lim \sup _{n \rightarrow \infty} \frac{\left\|u \varphi^{n}\right\|_{w}}{\left\|z^{n}\right\|_{v}}$.

Lemma 2.5 [22, Lemma 2.1] For $\alpha>0$, we have $\lim _{n \rightarrow \infty}(n+1)^{\alpha}\left\|z^{n}\right\|_{v_{\alpha}}=\left(\frac{2 \alpha}{e}\right)^{\alpha}$.

The following criterion for compactness follows from an easy modification of [25, Proposition 3.11]. Hence we omit the details.

Lemma 2.6 Let $0<\alpha, \beta<\infty$ and $T$ be a linear operator from $\mathcal{B}^{\alpha}$ to $\mathcal{B}^{\beta}$. Then $T: \mathcal{B}^{\alpha} \rightarrow \mathcal{B}^{\beta}$ is compact if and only if $T: \mathcal{B}^{\alpha} \rightarrow \mathcal{B}^{\beta}$ is bounded and for any bounded sequence $\left\{f_{k}\right\}_{k \in \mathbb{N}}$ in $\mathcal{B}^{\alpha}$ which converges to zero uniformly on compact subsets of $\mathbb{D},\left\|T f_{k}\right\|_{\mathcal{B}^{\beta}} \rightarrow 0$ as $k \rightarrow \infty$.

\section{Boundedness and essential norm of $C_{\varphi} D^{m}$}

In this section, we give other equivalent characterizations for the boundedness and the essential norm of the operator $C_{\varphi} D^{m}: \mathcal{B}^{\alpha} \rightarrow \mathcal{B}^{\beta}$ with $0<\alpha, \beta<\infty$.

Theorem 3.1 Let $0<\alpha, \beta<\infty, m \in \mathbb{N}$, and $\varphi \in S(\mathbb{D})$. Then the following statements are equivalent:

(a) $C_{\varphi} D^{m}: \mathcal{B}^{\alpha} \rightarrow \mathcal{B}^{\beta}$ is bounded. 
(b)

$$
\sup _{z \in \mathbb{D}} \frac{\left(1-|z|^{2}\right)^{\beta}\left|\varphi^{\prime}(z)\right|}{\left(1-|\varphi(z)|^{2}\right)^{\alpha+m}}<\infty
$$

(c)

$$
\sup _{n \geq 1} n^{\alpha+m}\left\|\varphi^{\prime} \varphi^{n-1}\right\|_{\nu \beta}<\infty
$$

Proof $(\mathrm{a}) \Rightarrow(\mathrm{b})$. Suppose that $C_{\varphi} D^{m}: \mathcal{B}^{\alpha} \rightarrow \mathcal{B}^{\beta}$ is bounded. Choose $f_{1}(z)=z^{m+1}$ and

$$
f_{w}(z)=\frac{1-|\varphi(w)|^{2}}{(1-\overline{\varphi(w)} z)^{\alpha}}, \quad w \in \mathbb{D}
$$

It is easy to verify that $f_{1} \in \mathcal{B}^{\alpha}$ and $f_{w} \in \mathcal{B}^{\alpha}$ for $w \in \mathbb{D}$. By $\left\|C_{\varphi} D^{m} f\right\|_{\beta} \preceq\|f\|_{\alpha}$ for $f \in \mathcal{B}^{\alpha}$, we obtain

$$
\sup _{z \in \mathbb{D}}\left(1-|z|^{2}\right)^{\beta}\left|\varphi^{\prime}(z)\right|<\infty
$$

and

$$
\sup _{z \in \mathbb{D}} \frac{\left(1-|z|^{2}\right)^{\beta}\left|\varphi^{\prime}(z)\right||\varphi(z)|^{m+1}}{\left(1-|\varphi(z)|^{2}\right)^{\alpha+m}}<\infty .
$$

Then it follows that

$$
\sup _{|\varphi(z)| \leq \frac{1}{2}} \frac{\left(1-|z|^{2}\right)^{\beta}\left|\varphi^{\prime}(z)\right|}{\left(1-|\varphi(z)|^{2}\right)^{\alpha+m}} \preceq \sup _{z \in \mathbb{D}}\left(1-|z|^{2}\right)^{\beta}\left|\varphi^{\prime}(z)\right|<\infty
$$

and

$$
\sup _{|\varphi(z)|>\frac{1}{2}} \frac{\left(1-|z|^{2}\right)^{\beta}\left|\varphi^{\prime}(z)\right|}{\left(1-|\varphi(z)|^{2}\right)^{\alpha+m}} \preceq \sup _{z \in \mathbb{D}} \frac{\left(1-|z|^{2}\right)^{\beta}\left|\varphi^{\prime}(z)\right||\varphi(z)|^{m+1}}{\left(1-|\varphi(z)|^{2}\right)^{\alpha+m}}<\infty .
$$

That is, (b) holds.

(b) $\Leftrightarrow(c)$. From Lemma 2.2, the condition (b) is a necessary and sufficient condition for the boundedness of weighted composition operator $\varphi^{\prime} C_{\varphi}: H_{v_{\alpha+m}}^{\infty} \rightarrow H_{v_{\beta}}^{\infty}$. Further by Lemma 2.4(a) and Lemma 2.5, the boundedness of the weighted composition operator $\varphi^{\prime} C_{\varphi}: H_{v_{\alpha+m}}^{\infty} \rightarrow H_{v_{\beta}}^{\infty}$ is equivalent to the following:

$$
\begin{aligned}
\sup _{n \geq 1} \frac{\left\|\varphi^{\prime} \varphi^{n-1}\right\|_{v_{\beta}}}{\left\|z^{n-1}\right\|_{v_{\alpha+m}}} & =\sup _{n \geq 1} \frac{n^{\alpha+m}\left\|\varphi^{\prime} \varphi^{n-1}\right\|_{v_{\beta}}}{n^{\alpha+m}\left\|z^{n-1}\right\|_{v_{\alpha+m}}} \\
& \asymp \sup _{n \geq 1} n^{\alpha+m}\left\|\varphi^{\prime} \varphi^{n-1}\right\|_{v_{\beta}}<\infty .
\end{aligned}
$$

(b) $\Rightarrow$ (a). Suppose (b) holds. For every $f \in \mathcal{B}^{\alpha}$, then it follows from Lemma 2.1 that

$$
\left\|C_{\varphi} D^{m} f\right\|_{\beta}=\sup _{z \in \mathbb{D}}\left(1-|z|^{2}\right)^{\beta}\left|f^{(m+1)}(\varphi(z)) \varphi^{\prime}(z)\right| \leq \sup _{z \in \mathbb{D}} \frac{\left(1-|z|^{2}\right)^{\beta}\left|\varphi^{\prime}(z)\right|}{\left(1-|\varphi(z)|^{2}\right)^{\alpha+m}}\|f\|_{\mathcal{B}^{\alpha}<\infty .}
$$


Moreover, $\left|C_{\varphi} D^{m} f(0)\right|=\left|f^{(m)}(\varphi(0))\right| \leq \frac{\|f\|_{\mathcal{B}^{\alpha}}}{\left(1-|\varphi(0)|^{2}\right)^{\alpha+m-1}}$. Thus $\left\|C_{\varphi} D^{m} f\right\|_{\mathcal{B}^{\beta}}<\infty$, and hence (a) holds.

\section{Remark 3.2}

(1) The relation (a) $\Leftrightarrow$ (b) was essentially proved in a very general result in [18]. For convenience of the reader, we sketch the proof in [18].

(2) One can easily see that

$$
\begin{aligned}
\sup _{n \in \mathbb{N}} n^{\alpha-1}\left\|C_{\varphi} D^{m} I_{n}(z)\right\|_{\beta} & =\sup _{n \geq m+1} n^{\alpha-1} n(n-1) \cdots(n-m)\left\|\varphi^{\prime} \varphi^{n-m-1}\right\|_{v_{\beta}} \\
& =\sup _{k \geq 1}(k+m)^{\alpha}(k+m-1) \cdots k\left\|\varphi^{\prime} \varphi^{k-1}\right\|_{v_{\beta}} \\
& \asymp \sup _{k \geq 1} k^{\alpha+m}\left\|\varphi^{\prime} \varphi^{k-1}\right\|_{v_{\beta}}=\sup _{n \geq 1} n^{\alpha+m}\left\|\varphi^{\prime} \varphi^{n-1}\right\|_{v_{\beta}} .
\end{aligned}
$$

Therefore, the characterizations for the boundedness of the operator $C_{\varphi} D^{m}$ in Theorem 3.1 are equivalent to that in Theorem A.

As an application of Theorem 3.1, we present an example of the bounded operator $C_{\varphi} D^{m}$, according to either (3.1) or (3.2).

Example 3.3 Let $\varphi(z)=z^{2}$ for $z \in \mathbb{D}$ and $\beta=\alpha+m$. Then we study the boundedness of $C_{\varphi} D^{m}: \mathcal{B}^{\alpha} \rightarrow \mathcal{B}^{\alpha+m}$. Firstly, by (3.1), it is clear that

$$
\sup _{z \in \mathbb{D}} \frac{\left(1-|z|^{2}\right)^{\alpha+m}\left|\varphi^{\prime}(z)\right|}{\left(1-|\varphi(z)|^{2}\right)^{\alpha+m}}=\sup _{z \in \mathbb{D}} \frac{\left(1-|z|^{2}\right)^{\alpha+m}|2 z|}{\left(1-|z|^{4}\right)^{\alpha+m}}<\infty .
$$

Secondly, by (3.2) we obtain

$$
\begin{aligned}
\sup _{n \geq 1} n^{\alpha+m}\left\|\varphi^{\prime} \varphi^{n-1}\right\|_{v_{\beta}} & =\sup _{n \geq 1} n^{\alpha+m}\left\|2 z z^{2(n-1)}\right\|_{v_{\beta}} \\
& =\sup _{n \geq 1} n^{\alpha+m} \sup _{z \in \mathbb{D}}\left(1-|z|^{2}\right)^{\alpha+m}\left|2 z z^{2(n-1)}\right| \\
& \preceq \sup _{n \geq 1} n^{\alpha+m} \sup _{x \in[0,1[}(1-x)^{\alpha+m} x^{n-\frac{1}{2}} \\
& =\sup _{n \geq 1} n^{\alpha+m}\left(1-\frac{n-\frac{1}{2}}{\beta+n-\frac{1}{2}}\right)^{\alpha+m}\left(\frac{n-\frac{1}{2}}{\beta+n-\frac{1}{2}}\right)^{n-\frac{1}{2}} \\
& =\sup _{n \geq 1}\left(\frac{\beta n}{\beta+n-\frac{1}{2}}\right)^{\alpha+m}\left(\frac{n-\frac{1}{2}}{\beta+n-\frac{1}{2}}\right)^{n-\frac{1}{2}}<\infty .
\end{aligned}
$$

From each of these conditions, one sees that $C_{\varphi} D^{m}: \mathcal{B}^{\alpha} \rightarrow \mathcal{B}^{\alpha+m}$ is bounded.

Next we estimate the essential norm of the operator $C_{\varphi} D^{m}: \mathcal{B}^{\alpha} \rightarrow \mathcal{B}^{\beta}$ for all $0<\alpha, \beta<$ $\infty$. Denote $\tilde{\mathcal{B}}^{\alpha}=\left\{f \in \mathcal{B}^{\alpha}: f(0)=0\right\}$. Let $D_{m+1}: \mathcal{B}^{\alpha} \rightarrow H_{v_{\alpha+m}}^{\infty}$ be defined by $D_{m+1} f=f^{(m+1)}(z)$. Then we have $\left\|D_{m+1} f\right\|_{v_{m+\alpha}} \asymp\|f\|_{\mathcal{B}^{\alpha}}$ for $f \in \tilde{\mathcal{B}}^{\alpha}$. Since $f^{(m+1)} \in H_{v_{\alpha+m}}^{\infty}$ when $f \in \mathcal{B}^{\alpha}$, and further by the equality $\left(C_{\varphi} D^{m} f\right)^{\prime}=\varphi^{\prime} f^{(m+1)}(\varphi)$ for all $f \in \mathcal{B}^{\alpha}$, it follows that

$$
\left\|C_{\varphi} D^{m}\right\|_{e, \tilde{\mathcal{B}}^{\alpha} \rightarrow \mathcal{B}^{\beta}} \preceq\left\|\varphi^{\prime} C_{\varphi}\right\|_{e, H_{\nu_{\alpha}+m}^{\infty} \rightarrow H_{\nu_{\beta}}^{\infty}}
$$


Thus we only need to estimate $\left\|\varphi^{\prime} C_{\varphi}\right\|_{e, H_{\nu^{\prime}+m}^{\infty} \rightarrow H_{\nu_{\beta}}^{\infty}}$ for the upper bound of the essential norm of $C_{\varphi} D^{m}$. It is obvious that every compact operator $T \in \mathcal{K}\left(\tilde{\mathcal{B}}^{\alpha}, \mathcal{B}^{\beta}\right)$ can be extended to a compact operator $K \in \mathcal{K}\left(\mathcal{B}^{\alpha}, \mathcal{B}^{\beta}\right)$. In fact, for every $f \in \mathcal{B}^{\alpha}, f-f(0) \in \tilde{\mathcal{B}}^{\alpha}$, and we can define $K(f):=T(f-f(0))+f(0)$, which is a compact operator from $\mathcal{B}^{\alpha}$ to $\mathcal{B}^{\beta}$, due to $K\left(f_{k}\right)$ has convergent subsequence when $\left\{f_{k}\right\}$ is a bounded sequence. In the following lemma we will use the compact operator $K_{r}$ defined on the space $\mathcal{B}^{\alpha}$ by $K_{r} f(z)=f(r z)$.

Lemma 3.4 If $0<\alpha, \beta<\infty$ and $C_{\varphi} D^{m}$ is a bounded operator from $\mathcal{B}^{\alpha}$ to $\mathcal{B}^{\beta}$, then

$$
\left\|C_{\varphi} D^{m}\right\|_{e, \tilde{\mathcal{B}}^{\alpha} \rightarrow \mathcal{B}^{\beta}}=\left\|C_{\varphi} D^{m}\right\|_{e, \mathcal{B}^{\alpha} \rightarrow \mathcal{B} \beta} .
$$

Proof Although the proof is similar to [20, Lemma 3.1], we will give all the details for convenience of the reader. It is obvious that

$$
\left\|C_{\varphi} D^{m}\right\|_{e, \tilde{\mathcal{B}}^{\alpha} \rightarrow \mathcal{B}^{\beta}} \leq\left\|C_{\varphi} D^{m}\right\|_{e, \mathcal{B}^{\alpha} \rightarrow \mathcal{B}^{\beta}} .
$$

Conversely, let $T \in K\left(\mathcal{B}^{\alpha}, \mathcal{B}^{\beta}\right)$ be given. Choose an increasing sequence $\left(r_{n}\right)_{n}$ in $(0,1)$ converging to 1 . We denote by $\mathcal{A}$ the closed subspace of $\mathcal{B}^{\alpha}$ consisting of all constant functions. Then we have

$$
\begin{aligned}
& \left\|C_{\varphi} D^{m}-T\right\|_{\mathcal{B}^{\alpha} \rightarrow \mathcal{B}^{\beta}}=\sup _{\|f\|_{\mathcal{B}^{\alpha} \leq 1}}\left\|C_{\varphi} D^{m}(f)-T(f)\right\|_{\mathcal{B}^{\beta}} \\
& \leq \sup _{\|f\|_{\mathcal{B}^{\alpha} \leq 1}}\left\|C_{\varphi} D^{m}(f-f(0))-\left.T\right|_{\tilde{\mathcal{B}}^{\alpha}}(f-f(0))\right\|_{\mathcal{B}^{\beta}} \\
& +\sup _{\|f\|_{\mathcal{B}^{\alpha} \leq 1}}\left\|C_{\varphi} D^{m}(f(0))-T(f(0))\right\|_{\mathcal{B}^{\beta}} \\
& \leq \sup _{g \in \tilde{\mathcal{B}}^{\alpha}}\left\|C_{\varphi} D^{m}(g)-\left.T\right|_{\tilde{\mathcal{B}}^{\alpha}}(g)\right\|_{\mathcal{B}^{\beta}}+\sup _{h \in \mathcal{A}}\left\|C_{\varphi} D^{m}(h)-\left.T\right|_{\mathcal{A}}(h)\right\|_{\mathcal{B}^{\beta}} .
\end{aligned}
$$

Hence

$$
\begin{aligned}
\inf _{T \in \mathcal{K}\left(\mathcal{B}^{\alpha}, \mathcal{B}^{\beta}\right)}\left\|C_{\varphi} D^{m}-T\right\|_{\mathcal{B}^{\alpha} \rightarrow \mathcal{B}^{\beta}} \leq & \inf _{T \in \mathcal{K}\left(\mathcal{B}^{\alpha}, \mathcal{B}^{\beta}\right)}\left\|C_{\varphi} D^{m}-\left.T\right|_{\tilde{\mathcal{B}}^{\alpha}}\right\|_{\tilde{\mathcal{B}}^{\alpha} \rightarrow \mathcal{B}^{\beta}} \\
& +\inf _{T \in \mathcal{K}\left(\mathcal{B}^{\alpha}, \mathcal{B}^{\beta}\right)}\left\|C_{\varphi} D^{m}-\left.T\right|_{\mathcal{A}}\right\|_{\mathcal{A} \rightarrow \mathcal{B}^{\beta}} \\
\leq & \left\|C_{\varphi} D^{m}\right\|_{e, \tilde{\mathcal{B}}^{\alpha} \rightarrow \mathcal{B}^{\beta}}+\lim _{n \rightarrow \infty}\left\|C_{\varphi} D^{m}\left(I-K_{r_{n}}\right)\right\|_{\mathcal{A} \rightarrow \mathcal{B}^{\beta}} .
\end{aligned}
$$

Since $C_{\varphi} D^{m}: \mathcal{B}^{\alpha} \rightarrow \mathcal{B}^{\beta}$ is bounded, it follows that

$$
\lim _{n \rightarrow \infty}\left\|C_{\varphi} D^{m}\left(I-K_{r_{n}}\right)\right\|_{\mathcal{A} \rightarrow \mathcal{B}^{\beta}} \leq C \lim _{n \rightarrow \infty}\left\|I-K_{r_{n}}\right\|_{\mathcal{A} \rightarrow \mathcal{B}^{\beta}}=0
$$

Thus we obtain $\left\|C_{\varphi} D^{m}\right\|_{e, \tilde{\mathcal{B}}^{\alpha} \rightarrow \mathcal{B}^{\beta}} \geq\left\|C_{\varphi} D^{m}\right\|_{e, \mathcal{B}^{\alpha} \rightarrow \mathcal{B}^{\beta}}$. The proof is finished.

Thus by Lemma 3.4 and (3.3) it follows that

$$
\left\|C_{\varphi} D^{m}\right\|_{e, \mathcal{B}^{\alpha} \rightarrow \mathcal{B}^{\beta}} \preceq\left\|\varphi^{\prime} C_{\varphi}\right\|_{e, H_{\nu_{\alpha}+m}^{\infty} \rightarrow H_{\nu_{\beta}}^{\infty}} .
$$


Theorem 3.5 Let $0<\alpha, \beta<\infty, m \in \mathbb{N}$, and $\varphi \in S(\mathbb{D})$. Suppose that $C_{\varphi} D^{m}: \mathcal{B}^{\alpha} \rightarrow \mathcal{B}^{\beta}$ is bounded. Then

$$
\begin{aligned}
\left\|C_{\varphi} D^{m}\right\|_{e, \mathcal{B}^{\alpha} \rightarrow \mathcal{B}^{\beta}} & \asymp \limsup _{n \rightarrow \infty} n^{\alpha+m}\left\|\varphi^{\prime} \varphi^{n-1}\right\|_{v_{\beta}} \\
& \asymp \limsup _{|\varphi(z)| \rightarrow 1} \frac{\left(1-|z|^{2}\right)^{\beta}\left|\varphi^{\prime}(z)\right|}{\left(1-|\varphi(z)|^{2}\right)^{\alpha+m}} .
\end{aligned}
$$

Proof If $\|\varphi\|_{\infty}<1$, then by [26, Lemma 3.1], the operator $u C_{\varphi}: \mathcal{B}^{\alpha} \rightarrow H_{\mu}^{\infty}$ is compact. The boundedness (compactness) of $C_{\varphi} D^{m}: \mathcal{B}^{\alpha} \rightarrow \mathcal{B}^{\beta}$ is equivalent to the boundedness (compactness) of $\varphi^{\prime} C_{\varphi}: \mathcal{B}^{\alpha+m} \rightarrow H_{\beta}^{\infty}$. In this case, all items in (3.5) are zero.

If $\|\varphi\|_{\infty}=1$, since $C_{\varphi} D^{m}: \mathcal{B}^{\alpha} \rightarrow \mathcal{B}^{\beta}$ is bounded, then the boundedness of $\varphi^{\prime} C_{\varphi}: H_{v_{\alpha+m}}^{\infty} \rightarrow$ $H_{v_{\beta}}^{\infty}$ follows from the proof in Theorem 3.1. Thus by (3.4), Lemma 2.4(b), and Lemma 2.5,

$$
\begin{aligned}
\left\|C_{\varphi} D^{m}\right\|_{e, \mathcal{B}^{\alpha} \rightarrow \mathcal{B}^{\beta}} & \preceq\left\|\varphi^{\prime} C_{\varphi}\right\|_{e, H_{v_{\alpha+m}}^{\infty} \rightarrow H_{v^{\prime}}^{\infty}}=\limsup _{n \rightarrow \infty} \frac{\left\|\varphi^{\prime} \varphi^{n-1}\right\|_{v_{\beta}}}{\left\|z^{n-1}\right\|_{v_{\alpha+m}}} \\
& =\limsup _{n \rightarrow \infty} \frac{n^{\alpha+m}\left\|\varphi^{\prime} \varphi^{n-1}\right\|_{v_{\beta}}}{\left\|z^{n-1}\right\|_{v_{\alpha+m}} n^{\alpha+m}} \asymp \limsup _{n \rightarrow \infty} n^{\alpha+m}\left\|\varphi^{\prime} \varphi^{n-1}\right\|_{v_{\beta}} .
\end{aligned}
$$

Since $\|\varphi\|_{\infty}=1$, we may choose a sequence $\left\{z_{k}\right\}_{k \in \mathbb{N}} \subset \mathbb{D}$ such that $\left|\varphi\left(z_{k}\right)\right| \rightarrow 1$ as $k \rightarrow \infty$. Define

$$
f_{k}(z)=\frac{1-\left|\varphi\left(z_{k}\right)\right|^{2}}{\left(1-\overline{\varphi\left(z_{k}\right)} z\right)^{\alpha}}, \quad k \in \mathbb{N} .
$$

It is easy to show that $f_{k} \in \mathcal{B}^{\alpha}$ and converges to zero uniformly on the compact subsets of $\mathbb{D}$ as $k \rightarrow \infty$. Moreover,

$$
f_{k}^{(m+1)}\left(\varphi\left(z_{k}\right)\right)=\frac{\alpha(\alpha+1) \cdots(\alpha+m)\left(\overline{\varphi\left(z_{k}\right)}\right)^{m+1}}{\left(1-\left|\varphi\left(z_{k}\right)\right|^{2}\right)^{\alpha+m}} .
$$

Then for every compact operator $T: \mathcal{B}^{\alpha} \rightarrow \mathcal{B}^{\beta}$, by Lemma 2.6, it follows that $\lim _{k \rightarrow \infty}\left\|T f_{k}\right\|_{\beta}=0$. Thus

$$
\begin{aligned}
\left\|C_{\varphi} D^{m}-T\right\|_{\mathcal{B}^{\alpha} \rightarrow \mathcal{B}^{\beta}} & \succeq \limsup _{k \rightarrow \infty}\left\|C_{\varphi} D^{m}\left(f_{k}\right)\right\|_{\beta}-\limsup _{k \rightarrow \infty}\left\|T f_{k}\right\|_{\beta} \\
& =\limsup _{k \rightarrow \infty}\left\|C_{\varphi} D^{m}\left(f_{k}\right)\right\|_{\beta} \\
& \geq \limsup _{k \rightarrow \infty}\left(1-\left|z_{k}\right|^{2}\right)^{\beta}\left|f_{k}^{(m+1)}\left(\varphi\left(z_{k}\right)\right) \varphi^{\prime}\left(z_{k}\right)\right| \\
& \succeq \limsup _{k \rightarrow \infty}\left(1-\left|z_{k}\right|^{2}\right)^{\beta} \frac{\left.\left|\varphi^{\prime}\left(z_{k}\right)\right| \varphi\left(z_{k}\right)\right|^{m+1}}{\left(1-\left|\varphi\left(z_{k}\right)\right|^{2}\right)^{\alpha+m}} \\
& =\limsup _{k \rightarrow \infty} \frac{\left(1-\left|z_{k}\right|^{2}\right)^{\beta}\left|\varphi^{\prime}\left(z_{k}\right)\right|}{\left(1-\left|\varphi\left(z_{k}\right)\right|^{2}\right)^{\alpha+m}} .
\end{aligned}
$$

Consequently,

$$
\left\|C_{\varphi} D^{m}\right\|_{e, \mathcal{B}^{\alpha} \rightarrow \mathcal{B}^{\beta}} \succeq \limsup _{|\varphi(z)| \rightarrow 1} \frac{\left(1-|z|^{2}\right)^{\beta}\left|\varphi^{\prime}(z)\right|}{\left(1-|\varphi(z)|^{2}\right)^{\alpha+m}}
$$


Since the operator $\varphi^{\prime} C_{\varphi}: H_{v_{\alpha+m}}^{\infty} \rightarrow H_{v_{\beta}}^{\infty}$ is bounded, then applying Lemma 2.3, Lemma 2.4(b), and Lemma 2.5, we get

$$
\begin{aligned}
\limsup _{|\varphi(z)| \rightarrow 1} \frac{\left(1-|z|^{2}\right)^{\beta}\left|\varphi^{\prime}(z)\right|}{\left(1-|\varphi(z)|^{2}\right)^{\alpha+m}} & \asymp\left\|\varphi^{\prime} C_{\varphi}\right\|_{e, H_{\nu_{\alpha}+m}^{\infty} \rightarrow H_{\nu_{\beta}}^{\infty}} \\
& =\limsup _{n \rightarrow \infty} \frac{\left\|\varphi^{\prime} \varphi^{n-1}\right\|_{\nu_{\beta}}}{\left\|z^{n-1}\right\|_{\nu_{\alpha+m}}} \asymp \limsup _{n \rightarrow \infty} n^{\alpha+m}\left\|\varphi^{\prime} \varphi^{n-1}\right\|_{\nu_{\beta}} .
\end{aligned}
$$

Thus

$$
\begin{aligned}
\limsup _{n \rightarrow \infty} n^{\alpha+m}\left\|\varphi^{\prime} \varphi^{n-1}\right\|_{\nu_{\beta}} & \succeq\left\|C_{\varphi} D^{m}\right\|_{e, \mathcal{B}^{\alpha} \rightarrow \mathcal{B}^{\beta}} \\
& \succeq \limsup _{|\varphi(z)| \rightarrow 1} \frac{\left(1-|z|^{2}\right)^{\beta}\left|\varphi^{\prime}(z)\right|}{\left(1-|\varphi(z)|^{2}\right)^{\alpha+m}} \\
& \asymp \limsup _{n \rightarrow \infty} n^{\alpha+m}\left\|\varphi^{\prime} \varphi^{n-1}\right\|_{\nu_{\beta}} .
\end{aligned}
$$

Hence

$$
\begin{aligned}
\left\|C_{\varphi} D^{m}\right\|_{e, \mathcal{B}^{\alpha} \rightarrow \mathcal{B}^{\beta}} & \asymp \limsup _{n \rightarrow \infty} n^{\alpha+m}\left\|\varphi^{\prime} \varphi^{n-1}\right\|_{\nu_{\beta}} \\
& \asymp \limsup _{|\varphi(z)| \rightarrow 1} \frac{\left(1-|z|^{2}\right)^{\beta}\left|\varphi^{\prime}(z)\right|}{\left(1-|\varphi(z)|^{2}\right)^{\alpha+m}} .
\end{aligned}
$$

This completes the proof.

\section{Remark 3.6}

(1) The relation $\left\|C_{\varphi} D^{m}\right\|_{e, \mathcal{B}^{\alpha} \rightarrow \mathcal{B}^{\beta}} \asymp \lim \sup _{|\varphi(z)| \rightarrow 1} \frac{\left(1-\left.|z|^{2}\right|^{\beta}\left|\varphi^{\prime}(z)\right|\right.}{\left(1-|\varphi(z)|^{2}\right)^{\alpha+m}}$ can be proved similarly to [26, Theorem 3.2]. Here we give a complete proof for the reader's convenience.

(2) Similar to Remark 3.2, one can get

$$
\limsup _{n \rightarrow \infty} n^{\alpha+m}\left\|\varphi^{\prime} \varphi^{n-1}\right\|_{v_{\beta}} \asymp \limsup _{n \rightarrow \infty} n^{\alpha-1}\left\|C_{\varphi} D^{m} I_{n}(z)\right\|_{\beta} .
$$

Therefore, the characterizations for the essential norms of the operator $C_{\varphi} D^{m}$ in Theorem 3.5 are equivalent to that in Theorem B.

The following corollary is an immediate consequence of Theorem 3.5.

Corollary 3.7 Let $0<\alpha, \beta<\infty, m \in \mathbb{N}$, and $\varphi \in S(\mathbb{D})$. Then the following statements are equivalent:

(a) $C_{\varphi} D^{m}: \mathcal{B}^{\alpha} \rightarrow \mathcal{B}^{\beta}$ is compact.

(b) $C_{\varphi} D^{m}: \mathcal{B}^{\alpha} \rightarrow \mathcal{B}^{\beta}$ is bounded and

$$
\limsup _{|\varphi(z)| \rightarrow 1} \frac{\left(1-|z|^{2}\right)^{\beta}\left|\varphi^{\prime}(z)\right|}{\left(1-|\varphi(z)|^{2}\right)^{\alpha+m}}=0 .
$$

(c) $C_{\varphi} D^{m}: \mathcal{B}^{\alpha} \rightarrow \mathcal{B}^{\beta}$ is bounded and

$$
\limsup _{n \rightarrow \infty} n^{\alpha+m}\left\|\varphi^{\prime} \varphi^{n-1}\right\|_{v_{\beta}}=0 .
$$




\section{Boundedness and essential norm of $D C_{\varphi}$}

In this section, the corresponding problems for the operator $D C_{\varphi}: \mathcal{B}^{\alpha} \rightarrow \mathcal{B}^{\beta}$ are considered. Let $u \in H(\mathbb{D})$, then for every $f \in H(\mathbb{D})$, define

$$
I_{u} f(z)=\int_{0}^{z} f^{\prime}(\zeta) u(\zeta) d \zeta, \quad J_{u} f(z)=\int_{0}^{z} f(\zeta) u^{\prime}(\zeta) d \zeta
$$

Then it follows that

$$
I_{\varphi^{\prime}}\left(\varphi^{n}\right)(z)=\int_{0}^{z}\left(\varphi^{n}\right)^{\prime}(\zeta) \varphi^{\prime}(\zeta) d \zeta, \quad J_{\varphi^{\prime}}\left(\varphi^{n-1}\right)(z)=\int_{0}^{z} \varphi^{n-1}(\zeta) \varphi^{\prime \prime}(\zeta) d \zeta .
$$

By an easy calculation, one can get

$$
\left(I_{\varphi^{\prime}}\left(\varphi^{n}\right)(z)\right)^{\prime}=n \varphi(z)^{n-1}\left(\varphi^{\prime}(z)\right)^{2}
$$

and

$$
\left(J_{\varphi^{\prime}}\left(\varphi^{n-1}\right)(z)\right)^{\prime}=\varphi(z)^{n-1} \varphi^{\prime \prime}(z) .
$$

In 2007, S Li and S Stević gave the following characterizations for the boundedness and compactness of the operator $D C_{\varphi}: \mathcal{B}^{\alpha} \rightarrow \mathcal{B}^{\beta}$.

Lemma 4.1 Let $\alpha, \beta>0$ and $\varphi \in S(\mathbb{D})$. Then the following statements hold:

(a) [4, Theorem 1] $D C_{\varphi}: \mathcal{B}^{\alpha} \rightarrow \mathcal{B}^{\beta}$ is bounded if and only if

$$
\sup _{z \in \mathbb{D}} \frac{\left|\varphi^{\prime}(z)\right|^{2}\left(1-|z|^{2}\right)^{\beta}}{\left(1-|\varphi(z)|^{2}\right)^{\alpha+1}}<\infty \quad \text { and } \quad \sup _{z \in \mathbb{D}} \frac{\left|\varphi^{\prime \prime}(z)\right|\left(1-|z|^{2}\right)^{\beta}}{\left(1-|\varphi(z)|^{2}\right)^{\alpha}}<\infty
$$

(b) $\left[4\right.$, Theorem 2] $D C_{\varphi}: \mathcal{B}^{\alpha} \rightarrow \mathcal{B}^{\beta}$ is compact if and only if $D C_{\varphi}: \mathcal{B}^{\alpha} \rightarrow \mathcal{B}^{\beta}$ is bounded,

$$
\lim _{|\varphi(z)| \rightarrow 1} \frac{\left|\varphi^{\prime}(z)\right|^{2}\left(1-|z|^{2}\right)^{\beta}}{\left(1-|\varphi(z)|^{2}\right)^{\alpha+1}}=0 \quad \text { and } \quad \lim _{|\varphi(z)| \rightarrow 1} \frac{\left|\varphi^{\prime \prime}(z)\right|\left(1-|z|^{2}\right)^{\beta}}{\left(1-|\varphi(z)|^{2}\right)^{\alpha}}=0
$$

First, we will give a brief proof of Theorem $\mathrm{C}$ as regards the bounded operator $D C_{\varphi}$ : $\mathcal{B}^{\alpha} \rightarrow \mathcal{B}^{\beta}$ for all $0<\alpha, \beta<\infty$.

Theorem 4.2 Let $0<\alpha, \beta<\infty$ and $\varphi \in S(\mathbb{D})$. Then $D C_{\varphi}: \mathcal{B}^{\alpha} \rightarrow \mathcal{B}^{\beta}$ is bounded if and only if

$$
\sup _{n \geq 1} n^{\alpha}\left\|I_{\varphi^{\prime}}\left(\varphi^{n}\right)\right\|_{\beta}<\infty \quad \text { and } \quad \sup _{n \geq 1} n^{\alpha}\left\|J_{\varphi^{\prime}}\left(\varphi^{n-1}\right)\right\|_{\beta}<\infty
$$

Proof Lemma 4.1 shows that $D C_{\varphi}$ maps $\mathcal{B}^{\alpha}$ boundedly into $\mathcal{B}^{\beta}$ if and only if (4.3) holds. On the other hand, Lemma 2.2 shows that (4.3) holds if and only if the weighted composition operators $\left(\varphi^{\prime}\right)^{2} C_{\varphi}$ maps $H_{v_{\alpha+1}}^{\infty}$ boundedly into $H_{v_{\beta}}^{\infty}$ and $\varphi^{\prime \prime} C_{\varphi}$ maps $H_{v_{\alpha}}^{\infty}$ boundedly into $H_{v_{\beta}}^{\infty}$, and hence it follows from Lemma 2.4(a) that (4.3) is equivalent to

$$
\sup _{n \geq 1} \frac{\left\|\left(\varphi^{\prime}\right)^{2} \varphi^{n-1}\right\|_{\nu_{\beta}}}{\left\|z^{n-1}\right\|_{\nu_{\alpha+1}}}<\infty \quad \text { and } \quad \sup _{n \geq 1} \frac{\left\|\varphi^{\prime \prime} \varphi^{n-1}\right\|_{\nu_{\beta}}}{\left\|z^{n-1}\right\|_{\nu_{\alpha}}}<\infty .
$$


Using Lemma 2.5, (4.1) and (4.2), then the boundedness of $D C_{\varphi}: \mathcal{B}^{\alpha} \rightarrow \mathcal{B}^{\beta}$ is equivalent to

$$
\sup _{n \geq 1} \frac{\left\|\left(\varphi^{\prime}\right)^{2} \varphi^{n-1}\right\|_{\nu_{\beta}} n^{\alpha+1}}{n^{\alpha+1}\left\|z^{n-1}\right\|_{\nu_{\alpha+1}}} \asymp \sup _{n \geq 1}\left\|\left(\varphi^{\prime}\right)^{2} \varphi^{n-1}\right\|_{\nu_{\beta}} n^{\alpha+1}=\sup _{n \geq 1} n^{\alpha}\left\|I_{\varphi^{\prime}}\left(\varphi^{n}\right)\right\|_{\beta}<\infty
$$

and

$$
\sup _{n \geq 1} \frac{n^{\alpha}\left\|\varphi^{\prime \prime} \varphi^{n-1}\right\|_{\nu_{\beta}}}{n^{\alpha}\left\|z^{n-1}\right\|_{\nu_{\alpha}}} \asymp \sup _{n \geq 1} n^{\alpha}\left\|\varphi^{\prime \prime} \varphi^{n-1}\right\|_{\nu_{\beta}}=\sup _{n \geq 1} n^{\alpha}\left\|J_{\varphi^{\prime}}\left(\varphi^{n-1}\right)\right\|_{\beta}<\infty .
$$

This completes the proof.

Now, we give a new proof of Theorem $D$ about the essential norm of $D C_{\varphi}: \mathcal{B}^{\alpha} \rightarrow \mathcal{B}^{\beta}$ for $0<\alpha, \beta<\infty$. We denote $\tilde{\mathcal{B}}^{\alpha}=\left\{f \in \mathcal{B}^{\alpha}: f(0)=0\right\}$. Let $D_{\alpha}: \mathcal{B}^{\alpha} \rightarrow H_{\nu_{\alpha}}^{\infty}$ and $S_{\alpha}: \mathcal{B}^{\alpha} \rightarrow H_{v_{\alpha+1}}^{\infty}$ be the first-order derivative operator and the second-order derivative operator, respectively. That is,

$$
D_{\alpha}(f)=f^{\prime}, \quad S_{\alpha}(f)=f^{\prime \prime} .
$$

By Lemma 2.1 we have

$$
\left\|D_{\alpha} f\right\|_{v_{\alpha}}=\|f\|_{\mathcal{B}^{\alpha}} \quad \text { and } \quad\left\|S_{\alpha} f\right\|_{v_{\alpha+1}} \asymp\|f\|_{\mathcal{B}^{\alpha}} \quad \text { for } f \in \tilde{\mathcal{B}}^{\alpha} .
$$

For $f \in \mathcal{B}^{\alpha}$, by Lemma 2.1, $f^{\prime \prime} \in H_{v_{\alpha+1}}^{\infty}$, and $f^{\prime} \in H_{v_{\alpha}}^{\infty}$. Then by the equation $\left(D C_{\varphi} f\right)^{\prime}=$ $f^{\prime \prime}(\varphi)\left(\varphi^{\prime}\right)^{2}+f^{\prime}(\varphi) \varphi^{\prime \prime}$, it follows that

$$
\left\|D C_{\varphi}\right\|_{e, \tilde{\mathcal{B}}^{\alpha} \rightarrow \mathcal{B}^{\beta}} \preceq\left\|\left(\varphi^{\prime}\right)^{2} C_{\varphi}\right\|_{e, H_{\nu_{\alpha+1}}^{\infty} \rightarrow H_{\nu_{\beta}}^{\infty}}+\left\|\varphi^{\prime \prime} C_{\varphi}\right\|_{e, H_{\nu_{\alpha}}^{\infty} \rightarrow H_{\nu_{\beta}}^{\infty}}
$$

Moreover, every compact operator $T \in \mathcal{K}\left(\tilde{\mathcal{B}}^{\alpha}, \mathcal{B}^{\beta}\right)$ can be extended to a compact operator $K \in \mathcal{K}\left(\mathcal{B}^{\alpha}, \mathcal{B}^{\beta}\right)$. Then similar to Lemma 3.4 , one can easily get

$$
\left\|D C_{\varphi}\right\|_{e, \tilde{\mathcal{B}}^{\alpha} \rightarrow \mathcal{B}^{\beta}}=\left\|D C_{\varphi}\right\|_{e, \mathcal{B}^{\alpha} \rightarrow \mathcal{B}^{\beta}} .
$$

Thus combining the above equation with (4.4), we obtain

$$
\left\|D C_{\varphi}\right\|_{e, \mathcal{B}^{\alpha} \rightarrow \mathcal{B}^{\beta}} \preceq\left\|\left(\varphi^{\prime}\right)^{2} C_{\varphi}\right\|_{e, H_{\nu_{\alpha+1}}^{\infty} \rightarrow H_{\nu_{\beta}}^{\infty}}+\left\|\varphi^{\prime \prime} C_{\varphi}\right\|_{e, H_{\nu_{\alpha}}^{\infty} \rightarrow H_{\nu_{\beta}}^{\infty}}
$$

According to (4.5), we only need to estimate the right two essential norms for the upper bound of the essential norm of $D C_{\varphi}: \mathcal{B}^{\alpha} \rightarrow \mathcal{B}^{\beta}$.

Theorem 4.3 Let $0<\alpha, \beta<\infty$ and $\varphi \in S(\mathbb{D})$. Suppose that $D C_{\varphi}: \mathcal{B}^{\alpha} \rightarrow \mathcal{B}^{\beta}$ is bounded. Then

$$
\left\|D C_{\varphi}\right\|_{e, \mathcal{B}^{\alpha} \rightarrow \mathcal{B}^{\beta}} \asymp \max \left\{\limsup _{n \rightarrow \infty} n^{\alpha}\left\|I_{\varphi^{\prime}}\left(\varphi^{n}\right)\right\|_{\beta}, \limsup _{n \rightarrow \infty} n^{\alpha}\left\|J_{\varphi^{\prime}}\left(\varphi^{n-1}\right)\right\|_{\beta}\right\} .
$$


Proof By Lemma 4.1(a) and Lemma 2.2, the boundedness of $D C_{\varphi}: \mathcal{B}^{\alpha} \rightarrow \mathcal{B}^{\beta}$ is equivalent to $\left(\varphi^{\prime}\right)^{2} C_{\varphi}: H_{v_{\alpha+1}}^{\infty} \rightarrow H_{v_{\beta}}^{\infty}$ and $\varphi^{\prime \prime} C_{\varphi}: H_{v_{\alpha}}^{\infty} \rightarrow H_{v_{\beta}}^{\infty}$ are bounded weighted composition operators.

The upper estimate. From Lemma 2.4(b) and Lemma 2.5, we obtain

$$
\begin{aligned}
\left\|\left(\varphi^{\prime}\right)^{2} C_{\varphi}\right\|_{e, H_{\nu_{\alpha+1}}^{\infty} \rightarrow H_{\nu_{\beta}}^{\infty}} & =\limsup _{n \rightarrow \infty} \frac{\left\|\left(\varphi^{\prime}\right)^{2} \varphi^{n-1}\right\|_{\nu_{\beta}}}{\left\|z^{n-1}\right\|_{\nu_{\alpha+1}}}=\limsup _{n \rightarrow \infty} \frac{n^{\alpha+1}\left\|\left(\varphi^{\prime}\right)^{2} \varphi^{n-1}\right\|_{\nu_{\beta}}}{n^{\alpha+1}\left\|z^{n-1}\right\|_{\nu_{\alpha+1}}} \\
& \asymp \limsup _{n \rightarrow \infty} n^{\alpha+1}\left\|\left(\varphi^{\prime}\right)^{2} \varphi^{n-1}\right\|_{\nu_{\beta}}=\limsup _{n \rightarrow \infty} n^{\alpha}\left\|I_{\varphi^{\prime}}\left(\varphi^{n}\right)\right\|_{\beta}, \\
\left\|\varphi^{\prime \prime} C_{\varphi}\right\|_{e, H_{\nu_{\alpha}}^{\infty} \rightarrow H_{\nu_{\beta}}} & =\limsup _{n \rightarrow \infty} \frac{\left\|\varphi^{\prime \prime} \varphi^{n-1}\right\|_{\nu_{\beta}}}{\left\|z^{n-1}\right\|_{\nu_{\alpha}}}=\limsup _{n \rightarrow \infty} \frac{\left\|\varphi^{\prime \prime} \varphi^{n-1}\right\|_{\nu_{\beta}} n^{\alpha}}{\left\|z^{n-1}\right\|_{\nu_{\alpha}} n^{\alpha}} \\
\asymp & \limsup _{n \rightarrow \infty} n^{\alpha}\left\|\varphi^{\prime \prime} \varphi^{n-1}\right\|_{\nu_{\beta}}=\limsup _{n \rightarrow \infty} n^{\alpha}\left\|J_{\varphi^{\prime}}\left(\varphi^{n-1}\right)\right\|_{\beta} .
\end{aligned}
$$

Then it follows from (4.5) that

$$
\left\|D C_{\varphi}\right\|_{e, \mathcal{B}^{\alpha} \rightarrow \mathcal{B}^{\beta}} \preceq \max \left\{\limsup _{n \rightarrow \infty} n^{\alpha}\left\|I_{\varphi^{\prime}}\left(\varphi^{n}\right)\right\|_{\beta}, \limsup _{n \rightarrow \infty} n^{\alpha}\left\|J_{\varphi^{\prime}}\left(\varphi^{n-1}\right)\right\|_{\beta}\right\} .
$$

The lower estimate. Let $\left\{z_{k}\right\}_{k \in \mathbb{N}}$ be a sequence in $\mathbb{D}$ such that $\left|\varphi\left(z_{k}\right)\right| \rightarrow 1$ as $k \rightarrow \infty$. Define

$$
\begin{aligned}
& f_{k}(z)=\frac{1-\left|\varphi\left(z_{k}\right)\right|^{2}}{\left(1-\overline{\varphi\left(z_{k}\right)} z\right)^{\alpha}}-\frac{\alpha}{\alpha+1} \frac{\left(1-\left|\varphi\left(z_{k}\right)\right|^{2}\right)^{2}}{\left(1-\overline{\varphi\left(z_{k}\right)} z\right)^{\alpha+1}}, \\
& g_{k}(z)=\frac{1-\left|\varphi\left(z_{k}\right)\right|^{2}}{\left(1-\overline{\varphi\left(z_{k}\right)} z\right)^{\alpha}}-\frac{\alpha}{\alpha+2} \frac{\left(1-\left|\varphi\left(z_{k}\right)\right|^{2}\right)^{2}}{\left(1-\overline{\varphi\left(z_{k}\right)} z\right)^{\alpha+1}} .
\end{aligned}
$$

We can easily show both $f_{k}$ and $g_{k}$ belong to $\mathcal{B}^{\alpha}$ and converge to zero uniformly on the compact subsets of $\mathbb{D}$ as $k \rightarrow \infty$. Moreover,

$$
\begin{aligned}
& f_{k}^{\prime}\left(\varphi\left(z_{k}\right)\right)=0, \quad f_{k}^{\prime \prime}\left(\varphi\left(z_{k}\right)\right)=\frac{-\alpha\left(\overline{\varphi\left(z_{k}\right)}\right)^{2}}{\left(1-\left|\varphi\left(z_{k}\right)\right|^{2}\right)^{\alpha+1}} ; \\
& g_{k}^{\prime}\left(\varphi\left(z_{k}\right)\right)=\frac{\alpha \overline{\varphi\left(z_{k}\right)}}{(\alpha+2)\left(1-\left|\varphi\left(z_{k}\right)\right|^{2}\right)^{\alpha}}, \quad g_{k}^{\prime \prime}\left(\varphi\left(z_{k}\right)\right)=0 .
\end{aligned}
$$

Then for every compact operator $T: \mathcal{B}^{\alpha} \rightarrow \mathcal{B}^{\beta}$, by Lemma 2.6 we obtain

$$
\begin{aligned}
& \left\|D C_{\varphi}-T\right\|_{\mathcal{B}^{\alpha} \rightarrow \mathcal{B}^{\beta}} \succeq \limsup _{k \rightarrow \infty}\left\|D C_{\varphi}\left(f_{k}\right)\right\|_{\beta} \geq \limsup _{k \rightarrow \infty}\left(1-\left|z_{k}\right|^{2}\right)^{\beta}\left|\frac{-\alpha\left(\varphi^{\prime}\left(z_{k}\right)\right)^{2}\left(\overline{\varphi\left(z_{k}\right)}\right)^{2}}{\left(1-\left|\varphi\left(z_{k}\right)\right|^{2}\right)^{\alpha+1}}\right|, \\
& \left\|D C_{\varphi}-T\right\|_{\mathcal{B}^{\alpha} \rightarrow \mathcal{B}^{\beta}} \succeq \limsup _{k \rightarrow \infty}\left\|D C_{\varphi}\left(g_{k}\right)\right\|_{\beta} \geq \limsup _{k \rightarrow \infty}\left(1-\left|z_{k}\right|^{2}\right)^{\beta}\left|\frac{\alpha \varphi^{\prime \prime}\left(z_{k}\right) \overline{\varphi\left(z_{k}\right)}}{(\alpha+2)\left(1-\left|\varphi\left(z_{k}\right)\right|^{2}\right)^{\alpha}}\right| .
\end{aligned}
$$


Since the weighted composition operators $\left(\varphi^{\prime}\right)^{2} C_{\varphi}: H_{v_{\alpha+1}}^{\infty} \rightarrow H_{v_{\beta}}^{\infty}$ and $\varphi^{\prime \prime} C_{\varphi}: H_{v_{\alpha}}^{\infty} \rightarrow H_{v_{\beta}}^{\infty}$ are bounded. Then applying Lemma 2.3, Lemma 2.4(b), and Lemma 2.5, it follows that

$$
\begin{aligned}
\left\|D C_{\varphi}\right\|_{e, \mathcal{B}^{\alpha} \rightarrow \mathcal{B}^{\beta}} & \succeq \limsup _{|\varphi(z)| \rightarrow 1}\left(1-|z|^{2}\right)^{\beta}\left|\frac{\left(\varphi^{\prime}(z)\right)^{2}}{\left(1-|\varphi(z)|^{2}\right)^{\alpha+1}}\right| \\
& \asymp\left\|\left(\varphi^{\prime}\right)^{2} C_{\varphi}\right\|_{e, H_{\nu_{\alpha+1}^{\infty}} \rightarrow H_{\nu_{\beta}}^{\infty}}=\limsup _{n \rightarrow \infty} \frac{\left\|\left(\varphi^{\prime}\right)^{2} \varphi^{n-1}\right\|_{\nu_{\beta}}}{\left\|z^{n-1}\right\|_{\nu_{\alpha+1}}} \\
& \asymp \limsup _{n \rightarrow \infty} n^{\alpha+1}\left\|\left(\varphi^{\prime}\right)^{2} \varphi^{n-1}\right\|_{\nu_{\beta}}=\limsup _{n \rightarrow \infty} n^{\alpha}\left\|I_{\varphi^{\prime}}\left(\varphi^{n}\right)\right\|_{\beta}, \\
\left\|D C_{\varphi}\right\|_{e, \mathcal{B}^{\alpha} \rightarrow \mathcal{B}^{\beta}} & \succeq \limsup _{|\varphi(z)| \rightarrow 1}\left(1-|z|^{2}\right)^{\beta}\left|\frac{\varphi^{\prime \prime}(z)}{\left(1-|\varphi(z)|^{2}\right)^{\alpha}}\right| \\
& \asymp\left\|\varphi^{\prime \prime} C_{\varphi}\right\|_{e, H_{\nu_{\alpha}}^{\infty} \rightarrow H_{\nu_{\beta}}^{\infty}}=\limsup _{n \rightarrow \infty} \frac{\left\|\varphi^{\prime \prime} \varphi^{n-1}\right\|_{\nu_{\beta}}}{\left\|z^{n-1}\right\|_{\nu_{\alpha}}} \\
& \asymp \limsup _{n \rightarrow \infty} n^{\alpha}\left\|\varphi^{\prime \prime} \varphi^{n-1}\right\|_{\nu_{\beta}}=\limsup _{n \rightarrow \infty} n^{\alpha}\left\|J_{\varphi^{\prime}}\left(\varphi^{n-1}\right)\right\|_{\beta} .
\end{aligned}
$$

Hence

$$
\left\|D C_{\varphi}\right\|_{e, \mathcal{B}^{\alpha} \rightarrow \mathcal{B}^{\beta}} \succeq \max \left\{\limsup _{n \rightarrow \infty} n^{\alpha}\left\|I_{\varphi^{\prime}}\left(\varphi^{n}\right)\right\|_{\beta}, \limsup _{n \rightarrow \infty} n^{\alpha}\left\|J_{\varphi^{\prime}}\left(\varphi^{n-1}\right)\right\|_{\beta}\right\}
$$

This completes the proof.

The following result is an immediate consequence of Theorem 4.3 and Lemma 4.1(b).

Corollary 4.4 Let $\alpha, \beta>0$ and $\varphi \in S(\mathbb{D})$. Then the following statements are equivalent:

(a) $D C_{\varphi}: \mathcal{B}^{\alpha} \rightarrow \mathcal{B}^{\beta}$ is compact.

(b) $D C_{\varphi}: \mathcal{B}^{\alpha} \rightarrow \mathcal{B}^{\beta}$ is bounded,

$$
\limsup _{n \rightarrow \infty} n^{\alpha}\left\|I_{\varphi^{\prime}}\left(\varphi^{n}\right)\right\|_{\beta}=0 \quad \text { and } \quad \limsup _{n \rightarrow \infty} n^{\alpha}\left\|J_{\varphi^{\prime}}\left(\varphi^{n-1}\right)\right\|_{\beta}=0
$$

\section{Competing interests}

The authors declare that they have no competing interests.

\section{Authors' contributions}

All authors conceived and drafted the manuscript, and read and approved the final manuscript.

\section{Author details}

'School of Mathematical Sciences, Tianjin Normal University, Tianjin, 300387, P.R. China. ${ }^{2}$ Department of Mathematics, Tianjin University, Tianjin, 300072, P.R. China.

\section{Acknowledgements}

The authors would like to thank the Journal Editorial Office and the referees for the useful comments and suggestions, which improved the presentation of this article. This work was supported in part by the National Natural Science Foundation of China (Grant Nos. 11201331; 11301373; 11401431).

Received: 29 September 2014 Accepted: 28 November 2014 Published: 12 Dec 2014

\section{References}

1. Hibschweiler, RA, Portnoy, N: Composition followed by differentiation between Bergman and Hardy spaces. Rocky Mt. J. Math. 35(3), 843-855 (2005)

2. Hyvärinen, $\mathrm{O}$, Nieminen, I: Weighted composition followed by differentiation between Bloch-type spaces. Rev. Mat. Complut. (2014). doi:10.1007/s13163-013-0138-y 
3. Krantz, SG, Stević, S: On the iterated logarithmic Bloch space on the unit ball. Nonlinear Anal. TMA 71, 1772-1795 (2009)

4. Li, S, Stević, S: Composition followed by differentiation between Bloch type spaces. J. Comput. Anal. Appl. 9, 195-206 (2007)

5. Li, S, Stević, S: Composition followed by differentiation from mixed norm spaces to $\alpha$-Bloch spaces. Sb. Math. 199(12), 1847-1857 (2008)

6. Li, S, Stević, S: Generalized composition operators on Zygmund spaces and Bloch type spaces. J. Math. Anal. Appl. $338,1282-1295(2008)$

7. Li, S, Stević, S: Composition followed by differentiation between $H^{\infty}$ and $\alpha$-Bloch spaces. Houst. J. Math. 35(1), 327-340 (2009)

8. Li, S, Stević, S: Products of composition and differentiation operators from Zygmund spaces to Bloch spaces and Bers spaces. Appl. Math. Comput. 217, 3144-3154 (2010)

9. Liang, YX, Zhou, ZH: Essential norm of product of differentiation and composition operators between Bloch-type spaces. Arch. Math. 100(4), 347-360 (2013)

10. Liang, YX, Zhou, ZH: New estimate of essential norm of composition followed by differentiation between Bloch-type spaces. Banach J. Math. Anal. 8, 118-137 (2014)

11. Ohno, S: Products of composition and differentiation on Bloch spaces. Bull. Korean Math. Soc. 46(6), 1135-1140 (2009)

12. Stević, S: Characterizations of composition followed by differentiation between Bloch-type spaces. Appl. Math. Comput. 218, 4312-4316 (2011)

13. Stević, S: On a new operator from the logarithmic Bloch space to the Bloch-type space on the unit ball. Appl. Math. Comput. 206, 313-320 (2008)

14. Stević, S: On a new integral-type operator from the Bloch spaces to Bloch-type spaces on the unit ball. J. Math. Anal. Appl. 354, 426-434 (2009)

15. Stević, S: Products of composition and differentiation operators on the weighted Bergman space. Bull. Belg. Math. Soc. Simon Stevin 16, 623-635 (2009)

16. Stević, S: Norm and essential norm of composition followed by differentiation from $\alpha$-Bloch spaces to $H_{\mu}^{\infty}$. Appl. Math. Comput. 207, 225-229 (2009)

17. Stević, S: Composition followed by differentiation from $H^{\infty}$ and the Bloch space to $n$-th weighted-type spaces on the unit disk. Appl. Math. Comput. 216, 3450-3458 (2010)

18. Stević, S: Weighted differentiation composition operators from $H^{\infty}$ and Bloch spaces to $n$-th weighted-type spaces on the unit disk. Appl. Math. Comput. 216, 3634-3641 (2010)

19. Wu, Y, Wulan, H: Products of differentiation and composition operators on the Bloch space. Collect. Math. 63, 93-107 (2012)

20. Esmaeili, K, Lindström, M: Weighted composition operators between Zygmund type spaces and their essential norms. Integral Equ. Oper. Theory 75(4), 473-490 (2013)

21. Zhu, KH: Operator Theory in Function Spaces. Dekker, New York (1990)

22. Hyvärinen, $\mathrm{O}$, Lindström, M: Estimates of essential norms of weighted composition operators between Bloch-type spaces. J. Math. Anal. Appl. 393, 38-44 (2012)

23. Contreras, MD, Hernández-Díaz, AG: Weighted composition operators in weighted Banach spaces of analytic functions. J. Aust. Math. Soc. A 69, 41-60 (2000)

24. Hyvärinen, O, Kemppainen, M, Lindström, M, Rautio, A, Saukko, E: The essential norm of weighted composition operators on weighted Banach spaces of analytic functions. Integral Equ. Oper. Theory 72, 151-157 (2012)

25. Cowen, CC, MacCluer, BD: Composition Operators on Spaces of Analytic Functions. CRC Press, Boca Raton (1995)

26. Stević, S: Essential norms of weighted composition operators from the $\alpha$-Bloch space to a weighted-type space on the unit ball. Abstr. Appl. Anal. 2008, Article ID 279691 (2008)

\section{Submit your manuscript to a SpringerOpen ${ }^{\odot}$ journal and benefit from:}

- Convenient online submission

Rigorous peer review

- Immediate publication on acceptance

- Open access: articles freely available online

- High visibility within the field

- Retaining the copyright to your article 